\title{
تأثير الطربقة المزدوجة على مهارة القراءة فى معهد الاستقامة المتكاملة الإسلامية بسيماوانج أومبيلين
}

\section{Septika Rudiamon}

\begin{abstract}
Institut Agama Islam Negeri Batusangkar e-mail: septikarudiamon02@gmail.com
\end{abstract}

\section{Akhyar Hanif}

Institut Agama Islam Negeri Batusangkar e-mail: akhyarhanif@iainbatusangkar.ac.id

\section{Amelia}

ملخص: المشكلة الأسـاسية لهذا البحث هي هل يوجد تأثير الطريقة المزدوجة على مهارة القراءة عند التلاميذ في معهد الاستقامة المتكاملة الإسلامية بسيماوانج أومبيلين. وغرضيه لمعرفة تأثير الطريقة المزدوجة على مهارة القراءة عند التلاميذ في هذا المعهد. قام الكاتب بمنهج تجريبي بتصميم كوّاسي التجريبي

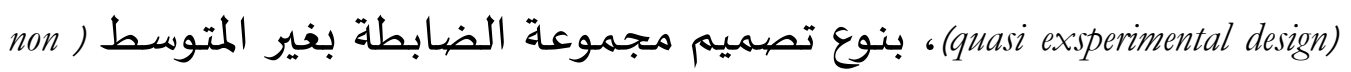
(R) Rondom (equivalent control group design الكاتب البيانات فحلّلها تحليلا إحصيائيا باستعمال الرمز test. أما النتيجة التي

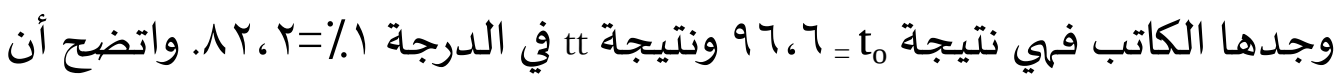

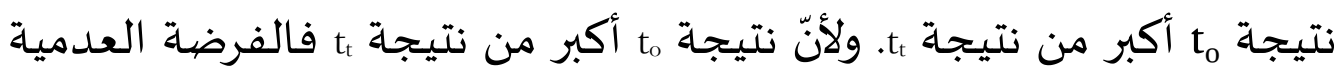
مردودة والفرضة التبادلية مقبولة. فالخلاصة أن الطريقة المزدوجة تؤثر على مهارة القراءة عند التلاميذ تأثيرا إيجابيا في هذا المعهد.

\section{الكلمات الأسـاسية: الطربقة المزدوجة، مهارة القراءة، اللغة العربية}

Abstract: The fundamental problem in this study is whether there is an effect of mixed method on the reading skills of Istiqomah Islamic Boarding School students or not. The aim is to determine the effect of mixed method on students' reading skills at this Islamic Borading school. This is an experimental study using a non equivalent control group quasi-experimental design. This type uses a non randomized system. After the data are obtained, then analyzed using the t- 
test. The result of data analysis describes that to $=6.96$ is higher than $t=2.82$ at the degree of $1 \%$. It indicates that the null hypothesis is rejected and the alternative hypothesis is accepted. It can be concluded that mixed method has a positive effect on students' reading skills in this Islamic Boarding School.

Key words: Mixed method, reading skill, arabic language

النصّ إلى آخره، ولا تكرّر قرائهها مرّة المقدمـة

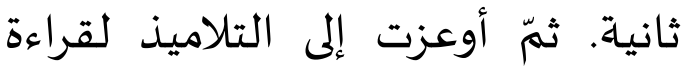
النصّ كلّه قراءة واحدة جماعاة. وبعد الند

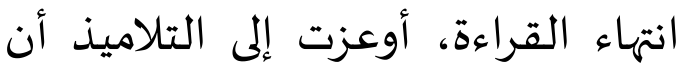
يطلب معنى النص، بعد ذلك بحثت

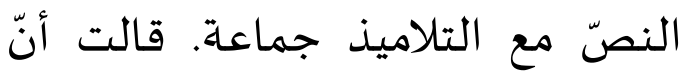
نتيجة بمعدّل تحديد معايير الكفاءة

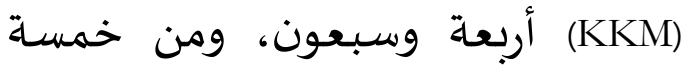
وأربعين تلميذا توجد سبعة عشرة تلميذا الذي وجد نتيجة فوق معدّل تحديد معايير الكفاءة (KKM)، وثمانية وعشرين تلميذا الذي وجد نتيجة تحت معدّل تحديد معايير الكفاءة (KKM). تمّثل هذه البيانات فيما يأتي (حاصل الملاحظة والمقابلة مع مدرّسة اللغة التة

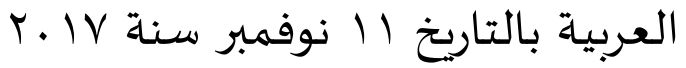
م في معهد الإستقامة المتكاملة الإسلامية بسيماوانج أومبيلين):

\begin{tabular}{|c|c|c|c|c|}
\hline غبر ناجح & ناجي & $\mu$ & نسل & رنم \\
\hline$\% 1 \xi=1 \xi$ & $\% \mu r=1$ & $r r$ & VIII a & 1 \\
\hline$\% \pi 1=1 \xi$ & $\%$ rq $=q$ & $\mu r$ & VIII b & $r$ \\
\hline
\end{tabular}

بناء على هذه المشكلة، قدّم

الكاتب علاجها باستعمال الطريقة بعد ما لاحظ الكاتب و قابل مدرّسة اللغة العربية يعني الأستاذة إثنيني في معهد الاستقامة المتكاملة الإسلامية بسيماوانج أومبيلين عن العنائن العملية التعليمية، فوجد الكاتب المشكلتين عن عملية تعليمية مهاراة القراءة، يعني: الأوّل أن يكون التلاميذ لم يستطعوا أن يقرؤوا نصوص اللغة الغوس العربية فصيحا صحيحا. فقد ظهرت من التلاميذ الأخطاء الكثيرة عن قراءة

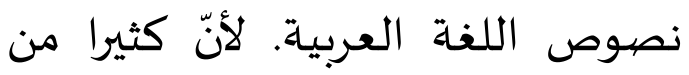

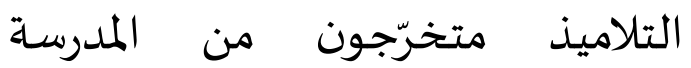

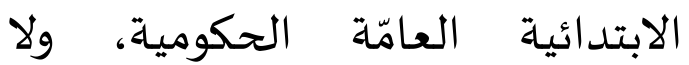
يدرسون اللغة العربية قبله في تلك المدرسة. إذن، هم يدرسون اللغة العربية لأوّل المرّة في هذا المعهد. والثَاني

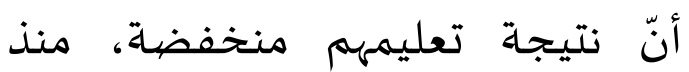
الامتحان الأوّل إلى الامتحان الثالث.

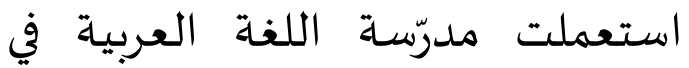
تعليم مهارة القراءة بطريقة السمعية

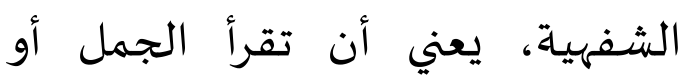
الفقرات من النصّ قراءة واحدة من أوّل النّل

91| Septika Rudiamon, Akhyar Hanif, Amelia 
حيث يعلم الكلمات صورة وصهوتا لينتقل تدريجيا إلى النظر في أجزائها كي يتمكّن من معرفهها ثانية وتهجئتها عند

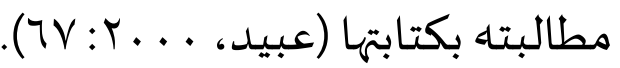
أما خطوات الطريقة المزدوجة يعني: () يقدم المعلم إلى الأطفال كلمات وجملا من المفردات التي يستعملونها ويفهمون معانيها ويتعرفونها كليا، فيميلون إلى قراءتها، وتتكرر هذا الكلمات والجمل حتّى تثبت في أذهانهم، فيستطيعون قراءتها بمجرد وقوع

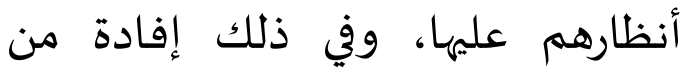

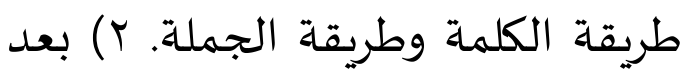
أن تثبت الكلمات والجمل في أذهان التلاميذ، تحلل الجملة إلى كلمات والكلمة إلى أحرف، فيتعلم التلاميذ التهال أصوات الحروف ويربطون بينها وبين

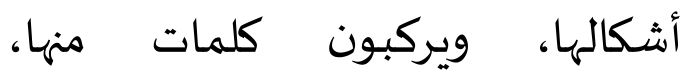
ويكسبون القدرة على قراءة كلمات جديدة، وفي ذلك إفادة من الطريقة الصوتية، ولكن البدء لا يكون بتعليم

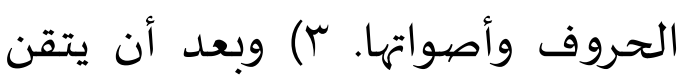
التلاميذ القراءة والكتابة، يتعلمون

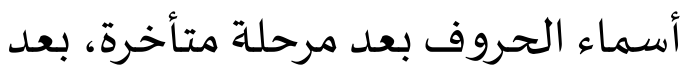

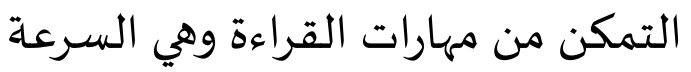
والفهم وجودة النطق وتعرف أشكال
المزدوجاة لرفع نتيجة تعليمهم في تعليم

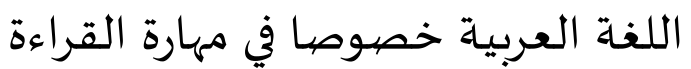

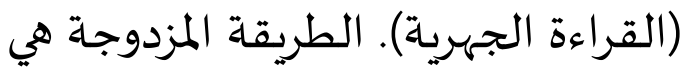

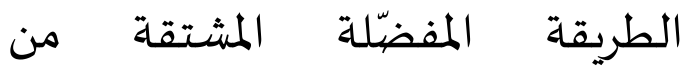
الطريقتين السابقتين والتي تجمع مزاياهما وتتجنّب عيوبها لكونها تجمع بين التحليل و والتركيب فسمّمّيت

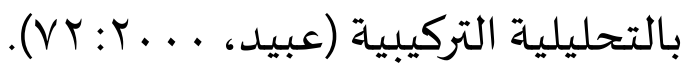
كما عرفنا، أنّ الطريقة المزدوجة تتكون الطريقة التركيبية والطريقة التحليلية وهما: أ) الطريقة التركيبية هي تبدأ

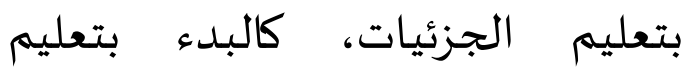

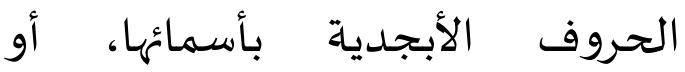
بأصواتها، ثمّ تنتقل بعد ذلك إلى تعليم المقاطع والكلمات والجمل التي تتألّف منها. أي أنّ هذه الطريقة تبدأ من أصغر وحدات ممكنة وتنتقل إلى الوحدات

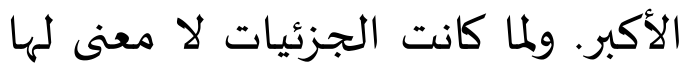

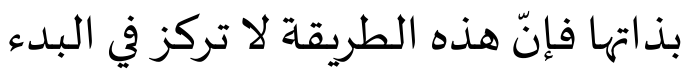
على المعنى (مدكور، ع191: 7צ (1). ب) الطريقة التحليلية (الكلّية) هي طريقة المدئ تسير بعكس الطريقة التركيبية، إذ تبدأ بتعليم الكلمة أو الجملة ثمّ الانتقال منهما إلى الحروف. أمّا أساس هذه الطريقة فهو معرفة الطفل لكثير من الطنائ الطيات الأشياء بأسمائها قبل دخوله المدرسة 
الرموز المطبوعة، ونقطها نطقا صحيحا (إذا كانت القراءة جهرية)، وفهمها. وعلى المئل هذا فهي تشمل التعرّف، وهو الاستجابة البصرية لما هو مكتوب،

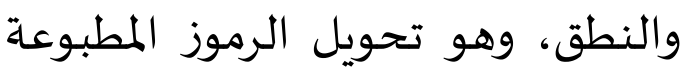
التي تمّت رؤيتها إلى أصوات ذات ذات معنى، المودو المطيوعه والفهم، أي ترجمة الرموز المدركة

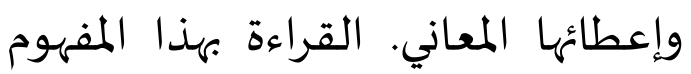
عملية معقّدة، ففيها تعرّف، وففيها

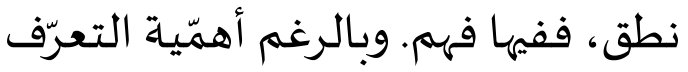

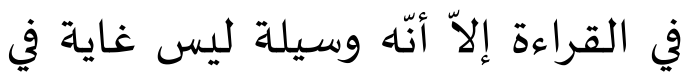
ذاته، فالتعرّف وسيلة للفهم (مدكور،

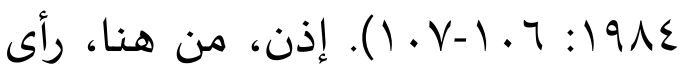
الكاتب، أنّ مهارة القراءة هي قدرة إدن هن التلاميذ في عملية التعرّف على الرموز المطبوعة، ونقطها نطقا صحيحا (إذا كانت القراءة جهرية)، وفهمها. فهي

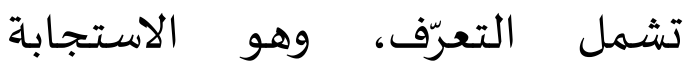
البصرية لما هو مكتوب، والنطق، وهو تحويل الرموز المطبوعة التي تمّت رؤيتها

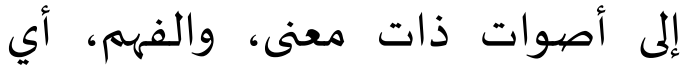
ترجمة الرموز المدركة وإعطائها المعاني. القراءة الجهرية هي القراءة بصوت مسموع، ونطق واضح صحيح لإكساب الطفل صعب النطق وإخراج الحروف من مخارجها الصحيحة، وهي
الحروف وأصهواتها، وفي ذلك إفادة من الطريقة الهجائية، ولكن البدء لا يكون بتعليم أسماء الحروف (الخليفة، .$(1+0-1 T \varepsilon: T . . \varepsilon$

أما مزايا الطريقة المزدوجة: أ) إن مادة القراءة مرتبطة ببيئتة التلاميذ، المردجها وكلماتها بسيطة من المفردات التي

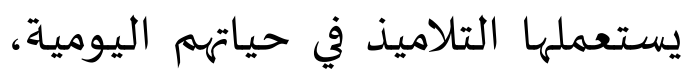
وبهذا فهي تثير اهتمامهم، فيكونون محبين للقراءة، ذوي ميول نحوها. ب) تساعد على أن يكسب الطفل، منذ البداية، القراءة على التعبير بما تقدمه لهاعل من مادة ذات وحدات فكرية متكاملة مترابطة. ج) هنتم اهتماما كبيرا بتعليم أشكال الحروف، وأصواتها، والحركات، والمد وتكسب الطفل المهارات الكتابية في الخط والإملاء. د) تعني، منذ البداية، بالتدريب على القراءة الصامتة، وتنهي لدى التلاميذ السرعة والدقة، والاعتماد

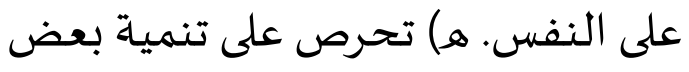
المهارات لدى المتعلم، كالميل إلى القراءة،

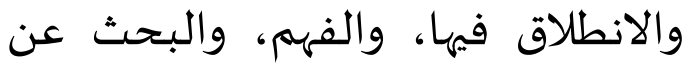

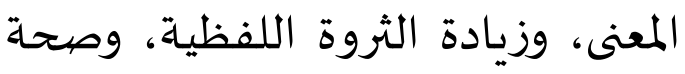

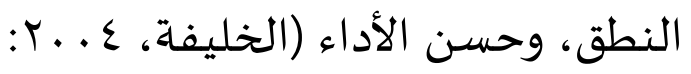
. (1) القراءة هي عملية التعرّف على 
أحسن وسيلة لإتقان النطق وإجادة 1 ).

إنّ الغرض من القراءة الجهرية

أن يمرن المدرّس التلاميذ على القراءة بصوت يسمع حتّى يستطيع أن يصلح لهم أخطاءهم في النطق والقراءة، ويعودهم السرعة بالتدريج، ليجيدوا

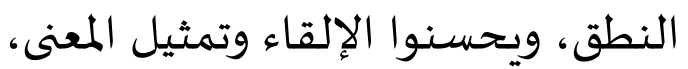

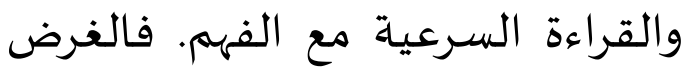
من القراءة الجهرية تعود التلاميذ حسن الإلقاء والتمثيل، وإظهار ما في القطعة من جمال حتّى يجد المستمعون لذة في

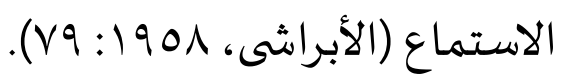

\section{طريقــة البحث}

أمّا نوع البحث الذي يستعمله

الكاتب فهي بحث تجريبي، ويستعمل

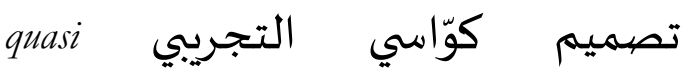
exsperimental design non equivalent ( الضابطة بغير المتوسط بوعة (R) group design control Rondom والطريقة المستعملة هي طريقة إحصائية لأنّه يبحث عن تأثير الطريقة

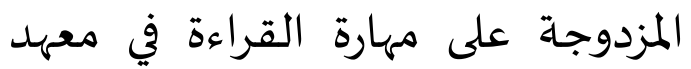
الإستقامة المتكاملة الإسلامية

بسيماوانج أومبيلين. أما مجتمع البحث المثله

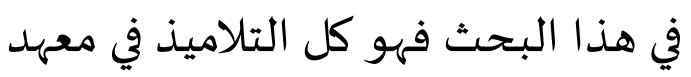

الأداء وتمثيل المعنى. وقد كانت فيما مضى موضع العناية في المدارس، ولكنّ ولمّن البحوث التي أجريت حول القراءة الصامتة، كشفت عن نتائج ترفح من

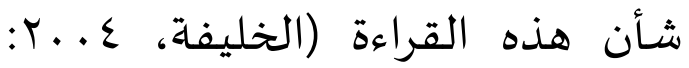

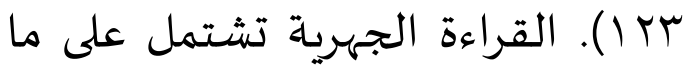

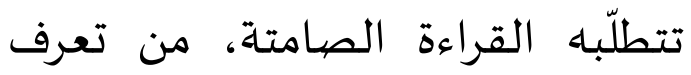
بصري للرموز الكتابية، وإدراك عقلي لمدلولاتها ومعانيها، وتزيد عليها التعبير لهري الشفهي عن هذه المدلولات والمعاني، بنطق الكلمات والجهر بها، وبذلك كانت

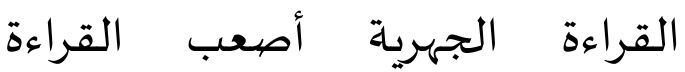

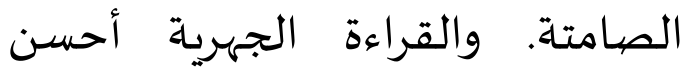
وسيلة لإتقان النطق، وإجادة الأداء، وتمثيل المعنى، ولاسيما في الصفوف

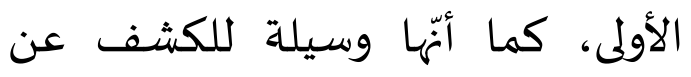
أخطاء التلاميذ في النطق، فيتسنى علاجها. وهي أيضا تساعد في الصفوف العليا على تذوّق الأدب بتعرّف نواحي الانسجام الصوتي والموسيقا اللفظية. وهي وسيلة لتشجيع التلاميذ ذوي الخوف والتهيب، وعلاج هذه الداء فيهم،

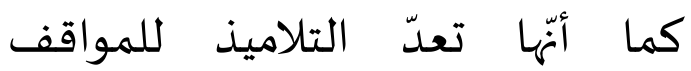

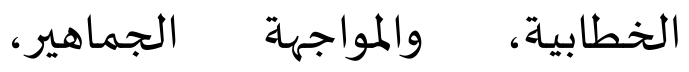
والحديث إلى الجماعة (الركابي، 1997 1: 
أمّا الغرض من امتحانين فهو لتأخذ الخلاصة بصحيحة وتمامة. ويستعمل

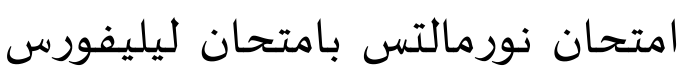
وامتحان هموجنيتس بامتحان (Liliefors) بارتليت (Bartlett). يستعمل امتحان نورمالتس البيانات بكيفية امتحان ليلفور (Liliefors) وامتحان Chi-Squere. لمعرفة نورمال أو غير نورمال من لمنان الامتحان يستعمل في إعطائه إلى عينة البحث، فيحلل بالحاسوب باسم SPSS 25. وامتحان هموجنيتس على فرقة باستعمال طريقة بارتليت (Bartlet). لمعرفة هموجنيتس أو غير هموجنيتس بارتليت من الامتحان يستعمل في إعطائه إلى عينة البحث، فيستعمل بالحاسوب باسم SPS 25 . تحليل البيانات هو سعي بكات الكاتب للحساب والبحث عن البيانات التي وجد كاتها في البحث، ويستطيع أن

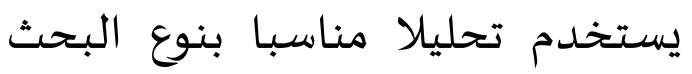
وأغراضها. ولتحليل الفرق بين نتيجة تعليم مهارة القراءة باستعمال الطريقة المزدوجة ونتيجة تعليم مهارة القراءة بغير استعمال الطريقة المزدوجة، يعلّم

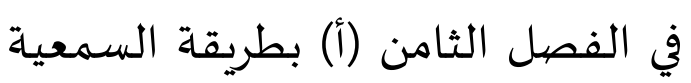
الشفهية فيقوم امتحان القراءة (القراءة الجهرية) ويعلّم في الفصل الثامن (ب)
الإستقامة المتكاملة الإسلامية بسيماوانج أومبيلين في المرحلة الثانوية،

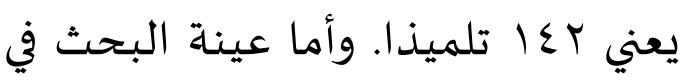
هذا البحث فهي التلاميذ في الفصل الفيل الفيل الثامن في المرحلة الثانوياة، يعني هـ تلميذا. وينقسم على الفرقتين، يعني في الفرقة (أ) عددهم اثنان وعشرون (YT) ويسمّى الفرقة الضابطة، والفرفة (ب)

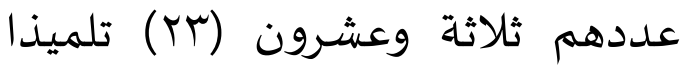
يسمّى الفرقة التجريبية. ويأخذ الكاتب عينة تجريبية باستعمال العشوائية التطبيقية (Random Sampling).

في هذا البحث يستعمل الكاتب تحليل ” "Tes “. أمّا الخطوات في البحث التجريبي فهي ما يلي:

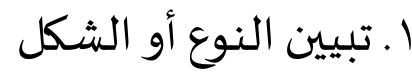

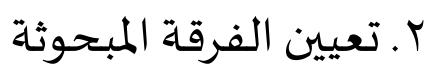

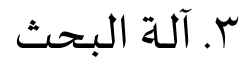
ع. ع. التجريبة 0. امتحان لشرط التحليل قبل أن يستعمل الكاتب امتحان الفرضة يحتاج إلى فحص البيانات لمتغيرات X و Y. في هذا الفحص يعمل امتحان نورمالتس (Uji Normalitas) وامتحان هموجنيهس (Uji Homogenitas). 
N N

T. يبحث الكاتب عن Standar eror dari mean of diference (SEMD) $\mathrm{SE}_{\mathrm{MD}}=\frac{\mathrm{SD}_{\mathrm{D}}}{\sqrt{\mathrm{N}}-1}$

V يبحث الكاتب عن t باستعمال الرمز:

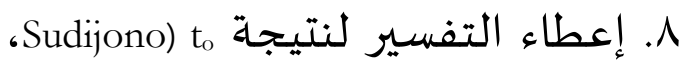
0 هذا البحث فهي بحث كمي و يكون تحليل البيانات التي استعملت فيه إحصيائيا وصفيا. جُمعت هذه البيانات بالاستبانة. قام الباحث بهذا البحث في جامعة باتوسنجكار الاسالامية الحكومية خاصة في المستوى الأول من قسم اللغة العربية. بلغ عدد الطلاب - اعتبارهم مجتمع البحث - مroاب طلاب في الفصل أ و r طاب في الفصل ب.

\section{نتائسجِ البحث ومبحثها}

جرب الكاتب الطريقة المزدوجة

على مهارة القراءة في الفرقة التجريبية فيقوم بالامتحان على (Eksperimentad) التلاميذ ووجد أقصى النتائج 90 وأدنى النتائج ع و و متوسطة النتيجة ع . 11. وهذه نتائج مهارة القراءة من سب تلميذا في الفصل الثامن، يعني نتائج مهارة
بالطريقة المزدوجة، فامتحنهم بالقراءة (القراءة الجهرية) أيضا، فوجد أساس المردوه النتيجتين، هما نتيـجة القراءة بالطريقة المزدوجة (X) ونتيجة القراءة بطريقة السمعية الشفهية (Y). ولتحليل النتيجتين يستعملهما الرمز لمعرفة النتيجتين، وسيجدل تأثير الطريقة المزدوجة في مهارة القراءة في معهد الإستقامة المتكاملة بسيماوانج أومبيلين وفي إنهاء البيانات فاستعمل الرمز ما يلي: $\mathrm{t}_{\mathrm{o}}=\underline{\mathrm{M}_{\mathrm{D}}}$ $\mathrm{SE}_{\mathrm{MD}}$

وأمّا خطوات إحصائيتها فهي ما يالي: ا. يبحث الكاتب عن تفريق بين النتيجتين باستعمال الرمز D=X-Y r. يبحث الكاتب عن نتيججة D حتّى يحصل على SD r. بحث الكاتب عن معدل التفريق

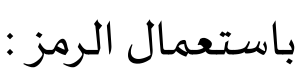
$\mathrm{M}_{\mathrm{D}}=\sum \frac{\mathrm{D}}{\mathrm{N}}$

ع. يضياعف الكاتب نتيدجة التفريق D ثمّ يبلغها حتّى يحصل نتيججة ه. يبحث الكاتب عن Defiasi Standar (SDD) dari Diference $\mathrm{SD}_{\mathrm{D}}=\sqrt{ } \sum \underline{\mathrm{D}^{2}}-\left(\sum \underline{\mathrm{D}^{2}}\right)$ 
الإستقامة المتكاملة الاسلامية

بسيماوانج أومبيلين. عادة المدرّسـة اللغة العربية تعلّم التلاميذ كمثل هذه الطريقة في مهارة القراءة. استعمل الكاتب الامتحان في الفرقة الضابطة لينظر نتيجة مهارة القراءة بطريقة السمعية الشفهية. من بيان نتيجة مهارة القراءة بالطريقة السمعية الشفهية،

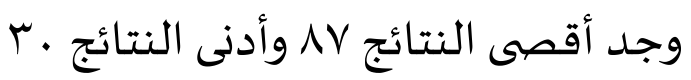
و متوسطة النتيجة م. 1.00. وهكذا نتيجة مهارة القراءة بطريقة السمعية الشفهية من الحاسوب فيما يلي:

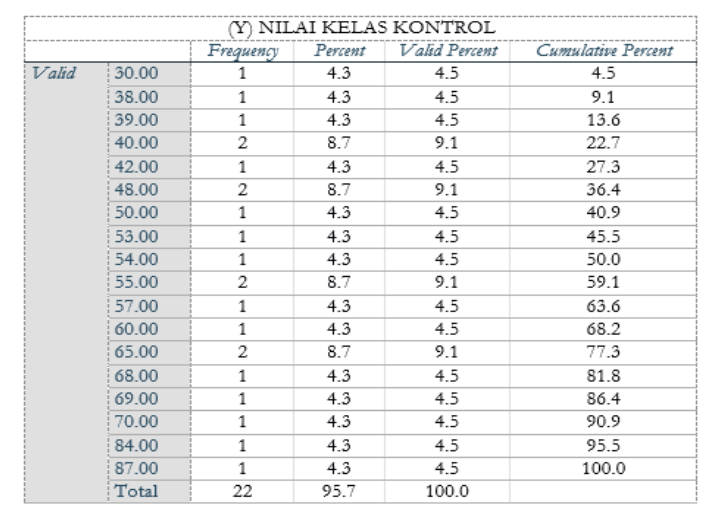
و هكذا نتيجة مهارة القراءة

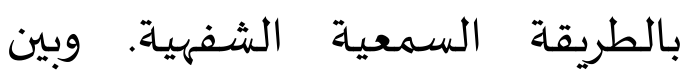
الجدول السابق عن نتائج مهارة القراءة من r T تلميذا بعدما حلل الكاتب بالحاسوب ووجد فياء أقصى النتائج

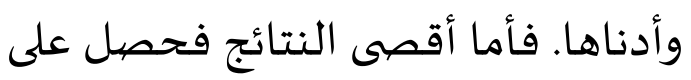

القراءة بالطريقة المزدوجة في الفرقة التجريبية. ونظر الكاتب نتيجة مهارة القراءة بالطريقة المزدوجة من

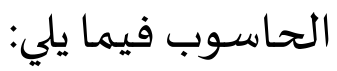

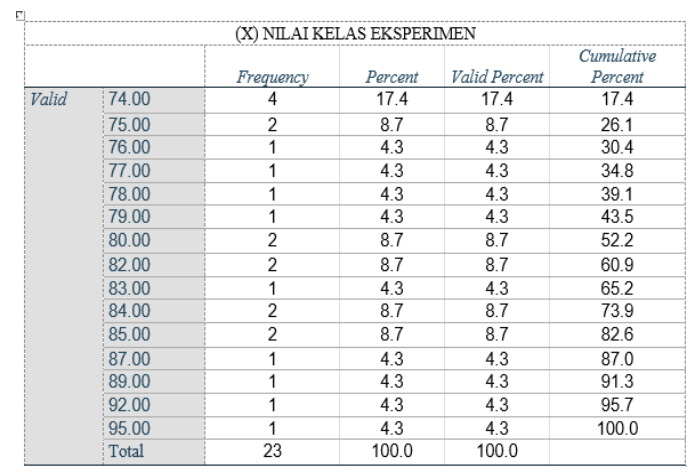

وهكذا نتيجة مهارة القراءة

بالطريقة المزدوجة. وبين الجدول

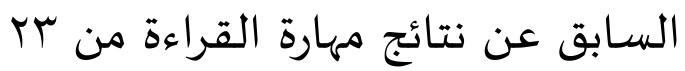
تلميذا بعدما حلل الكاتب بالحاسوب. ووجد فيه أقصى النتائج وأدناها. فأما باديا أقصى النتائج فحصل على تلميذ واحد

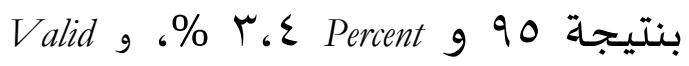
1.. Cumulative Percent 9 \% r \& \& Percent \%. وأدنى النتائج فحصل على و أربعة تلاميذا بنتيجة ع و V V ، IV Percent Cumulative g $\%$ \&。IV Valid Percentg \% ₹، IV Percent

\section{صفة الطريقة السمعية الشفهية} الطريقة السمعية الشفهية هي الطريقة التى تستعملها المدرّسـة في معهد 


\begin{tabular}{|c|c|c|c|c|c|}
\hline$D^{2}$ & $\mathrm{D}=(x-y)$ & r & $x$ & التلاديذ & رقمب \\
\hline 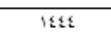 & $r A$ & $r \Lambda$ & rin & 1 & 1 \\
\hline ro & $\circ$ & 49 & $v_{\varepsilon}$ & $r$ & $r$ \\
\hline 1104 & $T s$ & \&. & $v_{\varepsilon}$ & $T$ & $r$ \\
\hline A1 & 9 & 40 & $v_{\varepsilon}$ & $\varepsilon$ & $\varepsilon$ \\
\hline $1 \leqslant$ & ir & u & A. & & \\
\hline ors & $\pi$ & of & w & 4 & \\
\hline ro & 0 & $\lambda V$ & at & 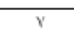 & $r$ \\
\hline$r v-\varepsilon$ & or & r. & AT & $A$ & $\lambda$ \\
\hline r. ro & so & $\varepsilon$ & 10 & 9 & 9 \\
\hline Tाo & ro & v. & 90 & 1. & \\
\hline 1998 & \& & st & $\Lambda \varepsilon$ & 11 & 11 \\
\hline $1 .$. & -1. & $\lambda \varepsilon$ & $v_{\varepsilon}$ & ir & ir \\
\hline ro.. & 0. & ri & 19 & it & $1 \pi$ \\
\hline sk & $\pi$ & or & $\mathrm{V}_{0}$ & 15 & 15 \\
\hline $\mathrm{rrq}$ & $\mathrm{rV}$ & « & $\mathrm{r}_{0}$ & 10 & 10 \\
\hline $1 . \Upsilon \varepsilon$ & $T r$ & 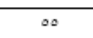 & $A Y$ & 14 & \\
\hline 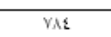 & is & or & 10 & iv & IY \\
\hline 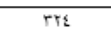 & in & 40 & AT & M & M \\
\hline$r r \xi$ & is & $\%$ & $v_{A}$ & 19 & 19 \\
\hline tho & ro & $\because$ & A. & $r$ & $r$. \\
\hline 1104 & $T \leqslant$ & 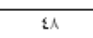 & Ar & $n$ & $\pi$ \\
\hline S\&1 & ria & 0. & va & $\pi$ & $\pi$ \\
\hline$Y .04$ & $\lambda \varepsilon$ & & $\Lambda \varepsilon$ & $\pi$ & $\pi$ \\
\hline$\sum \mathrm{D}^{2}=\mathrm{rq}(19$ & $\sum \mathrm{D}=4: \mathrm{v}$ & $Y=1 \pi \| \mathrm{V}$ & $\mathrm{x}=1,19 \mathrm{z}$ & & \\
\hline
\end{tabular}

وفي انتهاء البيانات السـابقات

استعمل الكاتب الرمز t test فحصل النتيجة 7.97.9. وبعد ذلك بحث عن df df = باستعمال الرمز (degree of freedom) df قبل ما فسر هذه النتيججة. فn-1

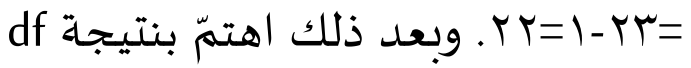
r = Tr في الجدول t في الدرجة 0 \% هي

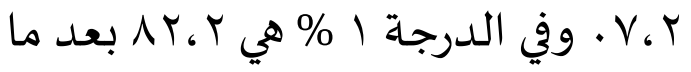

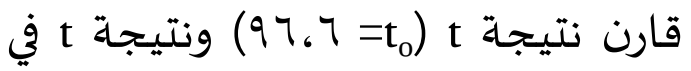

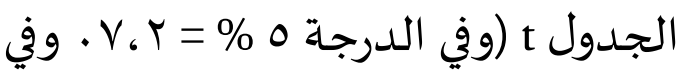

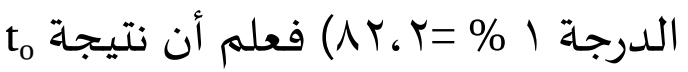

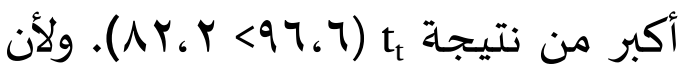
نتيججة t أكبر من نتيـجة th فالفرضية العدمية مردودة والفرضية التبادلية مقبولة، المراد بها أن هناك اختلاف بين نتيجة الامتحان الأول (نتيـجة مهارة القراءة بطريقة السمعية الشفهية)
تلميذ واحد بنتيججة NV و Percent ع ، \% \% Cumulative g \% 0 o \& Valid Percent g Percent على تلميذ واحد أيضيا بنتيجة . بـ ، \% \% 0 ¿ ¿ Cumulative Percent 9 امتحان الفرضة الفرض العملية التي جاءت من المشكلات القديمة فهي فيما يأتي: ا. يوجد تأثير إيجابي (+) الطريقة المزدوجـة في مهارة القراءة (H1). r. يوجد تأثير سلبيّ (-) الطريقة السمعيـة الشفهية في مهارة القراءة (H2). في هذه الإجراءات استعمل الكاتب التجربية بإجراء البحث التجربي في الفرقة الضيابطة فيه مهارة القراءة بطريقة السمعية الشفهية وفي الفرقة التجريبية بالطريقة المزدوجة. بعد ذلك استعمل الكاتب الامتحان على الفرقتين. ثم يقارن نتيـجة مهارة القراءة بالطريقة المزدوجة وطريقة السمعية الشفهية. بعدما قام الامتحان فحصل على نتيجاة مهارة القراءة بعد ما علّمههم بالطريقة المزدوجة. وبعد ذلك جمع في الجدول كما يالي: 
الأبراشى، م.ع. 1901. الطرق الخاصة في التوبية لتدريس اللغة العربية

والدين. القاهرة: مكتبة الأنجلو

المصرية.

الخليفة، ح. ج. ع...r. فصيول في تدريس اللغة العربية, إبتدائي- متئ

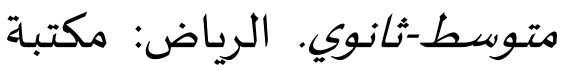

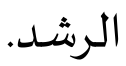

الركابي، ج. 1997. طرق تدريس اللغة العببية. سورية: دار الفكر.

السيد، م. أ. المواجز في طرائق تلدريس

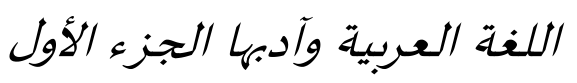
بيروت: مكتبة معهد العلوم

الإسلامية بإندونسيا.

إبراهيم، ح. 1919v. لإتجاهات المعاصرة. القاهرة: دار الفكر العربي.

إبراهيم، ع. ع. rأ197. الموجّه الفنى

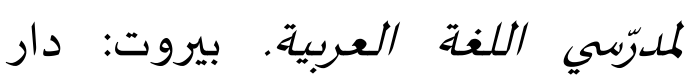

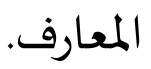

جابر، و. 1991 ـ أساليب تدريس اللغة

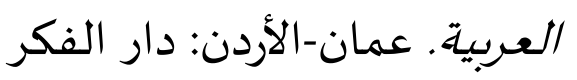
للنشر والتوزيع.

عبيد، ح. ر.ع. ر. .... .. طرق تلدريس
ونتيجة الامتحان الثانى (نتيجة مهارة القراءة بالطريقة المزدوجة).

\section{الخلاصية}

من نتيجة البحث السابق،

يستطيع الكاتب أن يأخذ الخلاصية أنّ النّا

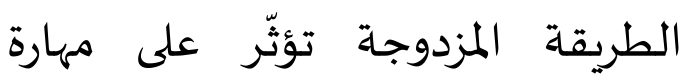
القراءة تأثيرا إيجابيّا في معهد الاستقامة تونة المتكاملة الإسلامية بسيماوانج أومبيلين. هناك الفرق بين نتيجة التلاميذ الذين

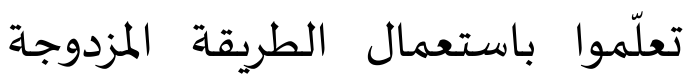
ونتيحة التلاميذ الذى تعلّم بطريقة السمعية الشفهية في مهارة القراءة في معهد الإستقامة المتكاملة الإسلامية

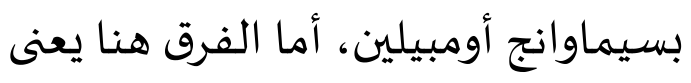

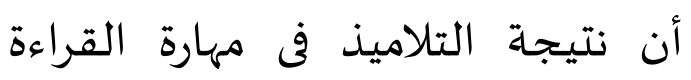

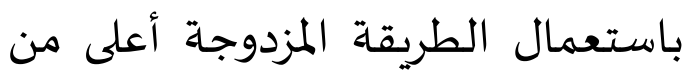

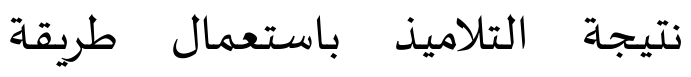
السمعية الشفهياة. وجد أقصى النتائج

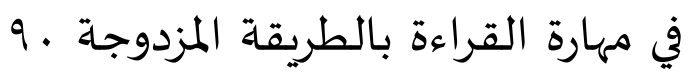

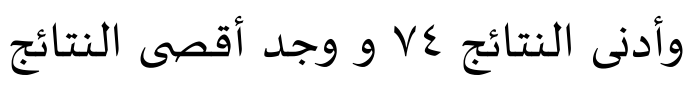
في مهارة القراءة بطريقة السمعية

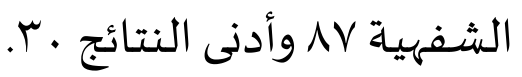


Sudijono, Anas. 2005. Pengantar Statistik Pendidikan. Jakarta: PT Raja Grafindo Persada.

Sugiyono. 2013. Metodologi Penelitian Kuantitatif dan Kualitatif dan $\mathrm{R}$ dan $\mathrm{D}$. Bandung: Alfabeta

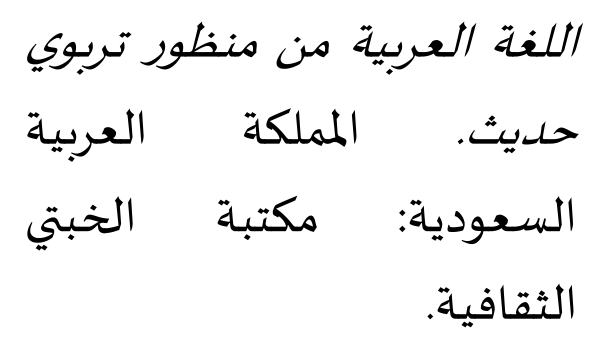

عثمان، ع. ر. أ. 1990 . مناهج البحث العملي وطرق كتابة الرسائل الجامعة. أفريقيا: جامعة أفريقيا

العالمياة.

عطا، إ. م. ج . .ب. الموجع في تلدرلس اللغتة العبربية. القاهرة: جامعة الملك عبد العزيز.

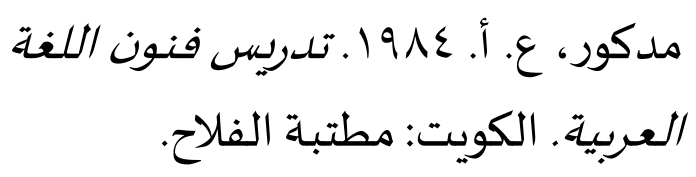

موسى، م. م. rا •r. الوافي في طق تملسيس اللغة العربية. الإمارات: دار ابن الجوزي.

هادي، ن. 11 . r. الموجه لتعليم المهارات اللغوية لغير الناطقين بها. مالانق: جامعة مولانا مالك إبراهيم الإسلامية الحكوميـة.

Hanafi, Abdul Halim. 2006. Metodologi Penelitian Bahasa. Batusangkar: STAIN Batusangkar Press.

Makmur, Ilzamuddin. 2006. Pilar-pilar Pemikiran Babasa dan Budaya. Jakarta: Diadit Media. 